\title{
Penetrative convection in a fluid saturated Darcy-Brinkman porous media with LTNE via internal heat source
}

https://doi.org/10.1515/nleng-2018-0053

Received March 19, 2018; revised July 18, 2018; accepted September 30, 2018.

\begin{abstract}
The present work involves the study of penetrative convection in an incompressible fluid-saturated porous media with local thermal non-equilibrium. The onset of convection evaluated linearly and nonlinearly for the system influenced by heat extraction and heat generation. Darcy-Brinkman law is employed to model the momentum equation and four type of internal heat generating function are considered which leads to thermoconvective instability within the fluid layer. Linear analysis carried out by using normal mode technique and nonlinear stability analysis has been done by energy method. Due to heat generation within the fluid layer and heat extraction through boundary, the subcritical instability may exist with higher possibility. Effects of various parameters as: inter-phase heat transfer parameter, DarcyBrinkman number, porosity-modified conductivity ratio, and heat parameter are explored on Darcy-Rayleigh number by Chebyshev pseudospectral method as numerical form and graphical form.
\end{abstract}

Keywords: heat source; energy method; Brinkman effect; Local thermal non-equilibrium

\section{Introduction}

Penetrative convection is a phenomenon which occurs due to convective instability arises by unstable equilibrium. When the region of unstable equilibrium is bounded by, the fluid in stable equilibrium, the convective motion penetrates into the neighboring region of stable equilibrium. There are two major reasons for this type of motion, one is

\footnotetext{
Amit Mahajan, Department of Applied Sciences, NIT Delhi, Narela110040, India, E-mail: amitmahajan2006@gmail.com

*Corresponding Author: Reena Nandal, Department of Applied Sciences, NIT Delhi, Narela-110040, India, E-mail:

reena12101@gmail.com
}

velocity and another is non-vanishing tangential stresses. Penetrative convection occurs in many natural occurring astrophysical and geophysical phenomenon. For example, in star convection, causing the granulation pattern observed on the surface, heating, and cooling of lakes, thermocline formation, and radioactive decay and much more as described by Matthews (1988). An extensive literature is available on penetrative convection. Penetrative convection due to the heat sink and nonlinear density in an isotropic porous layer is examined by Carr \& Putter (2003). In thermo-convective instability, radiation also plays an important role in a stratified system. Earth's atmosphere is a good example to understand the phenomena of thermo-convective instability. The solar radiations heat up the surface and air very near to ground become warmer and lighter than upper heavy air and this creates gravitational instability. As convection starts, the warm air penetrates in stable regions. Effect of radiative heating through the lower boundary for a non-Boussinesq fluid layer is determined by Straughan (1991). An experimental study is done by Krishnamurti (1997) to observe the convective instability in a water layer with thymol blue in presence of a chemical concentration-dependent heat source. To understand the stability properties and scaling laws associated with a radiative-convective model, a study is presented by Larson (2000). Hill (2004) performed the stability analysis for a fluid layer which is thermo-convectively unstable due to concentration based internal heat source and absorption of radiations. When a water pool absorbs the solar radiations, it gets heated up and rises to the surface. In this process, it loses its heat to the atmosphere and remains at atmospheric temperature. To improve the ability of solar ponds to store the solar radiation, a stability analysis is done by Hill \& Carr (2013a, 2013b).

In porous media convective heat transfer process is found to be having important applications in thermal insulation of building, drying process, geophysical systems, petroleum resources, heat exchangers, chemical reactors, nuclear waste etc. The literature reveals that heat transfer processes in porous medium gained a considerable momentum due to its applicability in various fields. The book by Nield \& Bejan (2006) provides the diverse knowl- 
edge of this topic. For the study of fluid flow behavior in porous media Darcy's law is employed but it has limitations in several aspects. It is valid essentially for creeping flow through a long and uniform porous medium of low hydraulic conductivity. But in many technological problems, the fluid flow is very fast and Darcy's law is not enough to describe the behavior of fluid flow. For such situation, Brinkman (1947) suggested that the classical fractional term must be added to model the momentum equation, and this model known as Brinkman's model. An enormous work is present in the literature which provides a good understanding of the topic [Postelnicu (2008), Sunil et al. (2010), Shivakumara \& Dhananjaya (2015), Umavathi et al. (2017)]. Rees (2002) investigated that how highly porous boundaries (Brinkman) affect convection of Boussinesq fluid in a Darcy-porous medium. Very recently effect of a variety of internal heat generating source is observed by Nandal \& Mahajan (2017) for a fluid-saturated DarcyBrinkman porous media, to examine the effect of heat generation on the stability of the system.

Many investigations conducted on the flow in a porous media hypothesized that the solid matrix and fluid within the pore are in local thermal equilibrium. From an application point of view, the processes involving rapid heating and modeling of nuclear reactor, in fluid and solid matrix there exist temperature gradient so it is very uncertain the existence of local thermal equilibrium for such situations. A huge literature on local thermal non-equilibrium is provided by Straughan (2015). The onset of Darcy-Bénard convection using a local thermal non-equilibrium model is investigated by Banu \& Rees (2002). Wang et al. (2014) analyzed the effect of thermal radiations within a porous medium incorporated with local thermal non-equilibrium, for a specific application of solar receivers. The thermoconvective stability in porous media with throughflow and local thermal non-equilibrium with heterogeneity is investigated by Nield \& Kuznetsov (2015).

Several studies have considered the onset of penetrative convection in porous media with LTE but due attention has not been given to such studies in the context of LTNE. Such studies find its relevance in many technological and experimental problems. Intend of this work is stability analysis of a fluid layer saturated porous media with LTNE. It is considered that heat generation within the fluid layer and heat extraction through lower boundary induces the thermo-convective instability in the fluid layer. Four heat generating functions are considered for the stability analysis. Nonlinear stability results are compared with the linear instability results to observe whether there exists any subcritical instability or not. Effects of inter-phase heat transfer, Brinkman effect, internal heating and effects of heat extraction through the boundary, diffusivity and thermal conductivity of both the phases are considered to see the variation in critical Darcy-Rayleigh number by linear and nonlinear analysis and the corresponding values are achieved using Chebyshev tau-QZ method and are presented by graphs and numerical results.

\section{Mathematical formulation}

Here we consider an infinite layer of a thickness $d$ of an incompressible fluid saturated in a homogeneous porous media. The fluid assumes to confined between the layer $z=0, d$ and respective boundaries conditions are $\frac{\partial T_{s}}{\partial z}=$ $\frac{\partial T_{f}}{\partial z}=y$ at $z=0$ and $T_{S}=T_{f}=T_{U}$ at $z=d$, where $y$ represents the rate of heat extraction through the lower boundary. Boundaries are assumed impermeable and conducting with gravity $\mathbf{g}$ acts in negative $z$-direction and the Boussinesq approximation is employed to drive the density variation. Further, on the momentum equation modified Darcy model is employed and solid-fluid phases are with LTNE. Hence for the proposed model system of flow governing equations (Gasser \& Kazimi 1976; Straughan 2015) is given as:

Momentum equation:

$$
\frac{\rho_{f}}{\varepsilon} \frac{\partial \mathbf{q}}{\partial t}=-\nabla P-\frac{\mu_{f}}{K} \mathbf{q}+\mu_{f}^{\prime} \nabla^{2} \mathbf{q}+\rho_{f} \alpha T_{f} \mathbf{g} \hat{k}
$$

Mass balance equation:

$$
\nabla \cdot \mathbf{q}=0
$$

Temperature equation (fluid phase):

$$
\varepsilon \frac{\partial T_{f}}{\partial t}+\mathbf{q} \cdot \nabla T_{f}=\varepsilon \kappa \nabla^{2} T_{f}-\frac{h}{(\rho c)_{f}}\left(T_{s}-T_{f}\right)+Q_{f}
$$

Temperature equation (solid phase):

$$
(1-\varepsilon)(\rho c)_{s} \frac{\partial T_{s}}{\partial t}=(1-\varepsilon) k_{s} \nabla^{2} T_{s}+h\left(T_{s}-T_{f}\right)+Q_{s}
$$

where q, $\rho_{f}, c_{f}, \rho_{s}, c_{s}, P, \mu_{f}, \mu_{f}^{\prime}, t, \varepsilon, k_{f}, k_{s}, \kappa$ and Kare filter velocity, density of the fluid, heat capacity of fluid, density of solid matrix, heat capacity of solid matrix, pressure, the dynamic viscosity of fluid, effective viscosity of fluid, time, porosity, the thermal conductivity of fluid, the thermal conductivity of solid, thermal diffusivity of fluid, and permeability, respectively.

Now, the steady state solution of governing equations is as follows:

$$
\mathbf{q}_{\mathbf{b}}=\mathbf{0}, P_{b}=P(z), \rho_{b}=\rho(z), T_{b}=T(z) .
$$


here, the basic state followed by subscript ' $b$ '. The temperature of the solid and fluid phase at the basic state is $T_{f}=T_{s}$. From the equation (3) and (4) it is found that $T_{f}^{\prime \prime}=\frac{Q_{f}}{\varepsilon \kappa}$ and $T_{s}^{\prime \prime}=\frac{Q_{s}}{(1-\varepsilon) k_{s}}$, so the internal heat generation is related by the relation $\frac{Q_{f}}{\varepsilon \kappa}=\frac{Q_{s}}{(1-\varepsilon) k_{s}}$. This relation allows to define the internal heat generation function in one manner and here we restrict ourselves to $Q_{f}$.

In a thermodynamical system, internal heatgenerating functions are of many types. Roberts (1967) dealt with a constant heat source whereas Straughan (1991) examined the onset of convection in a horizontal layer in the context of three internal heat generating sources, i.e., constant, exponential and non-uniform heat source. In our study the following four type heatgenerating functions, i.e. constant, linearly increasing, decreasing and non-uniform (Straughan 2004), are considered:

Case A: $Q_{f}=Q$

Case B: $Q_{f}=Q\left(\frac{1}{2}+\frac{z}{d}\right)$

Case C: $Q_{f}=Q\left(2+\frac{3 z^{2}}{2 d^{2}}-3 \frac{z}{d}\right)$

Case D: $Q_{f}=Q\left(1+\sin \frac{2 \pi z}{d}+\sin \frac{4 \pi z}{d}\right)$

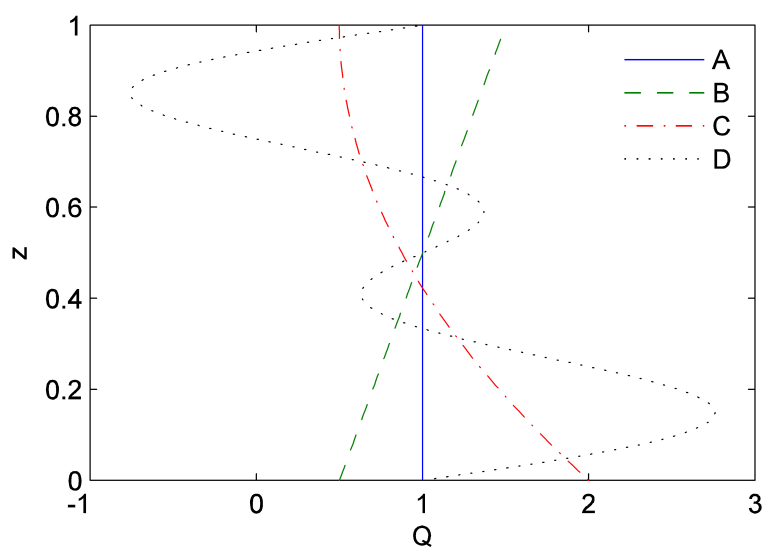

Fig. 1: Profile of various heat sources within the fluid layer

Now, the basic temperature function $T_{b}$ for the four cases is given as:

$$
\begin{aligned}
& T_{b}= \\
& \left\{\begin{array}{l}
-\frac{Q}{2 \varepsilon \kappa}\left(z^{2}-d^{2}\right)+y z+T_{U}-y d \text { case } A \\
-\frac{Q}{\varepsilon \kappa}\left(\frac{z^{3}}{6 d}+\frac{z^{2}}{4}-\frac{5 d^{2}}{12}\right)+y z+T_{U}-y d \text { case } B \\
-\frac{Q}{\varepsilon \kappa}\left(\frac{z^{4}}{8 d^{2}}-\frac{z^{3}}{2 d}+z^{2}-\frac{5 d^{2}}{8}\right)+y z+T_{U}-y d \text { case } C \\
-\frac{Q}{\varepsilon \kappa}\left(\frac{z^{2}}{2}-\frac{d^{2}}{4 \pi^{2}} \sin \frac{2 \pi z}{d}-\frac{d^{2}}{16 \pi^{2}} \sin \frac{4 \pi z}{d}+\frac{3 d}{4 \pi} z-\frac{d^{2}}{2}-\frac{3 d^{2}}{4 \pi}\right) \\
+y z+T_{U}-y d \text { case } D
\end{array}\right.
\end{aligned}
$$

To find out penetrative convection in the fluid layer, extremum of density is required and this can be followed from $T_{b}$. It, in turn, provide the range of heat parameter $\xi$ [defined later in (12)] to observe the effect of penetrative convection. The range of heat parameter obtained from maximization or minimization of the density function is given by $(0,1)$ for all cases and beyond this range, convection is due to buoyancy forces. Figure 2 displayed the profile of basic temperature functions along with the fluid layer. It can be seen from figure 2 that the small values of $\xi$ creates a high-temperature difference across the fluid layer, which enhances the convective movement from lower to upper parts of the fluid layer, and as the values of $\xi$ increases, the temperature difference becomes small and the fluid layer achieves the stability in the lower parts. To check the system stability we introduced the following perturbed quantities:

$$
\begin{aligned}
& \mathbf{q}=\mathbf{q}_{b}+\mathbf{q}^{\prime}, P=P_{b}+P^{\prime}, T_{f}=T_{b}+\theta, T_{s}=T_{b}+\phi, \\
& \rho=\rho_{b}+\rho^{\prime},
\end{aligned}
$$

where $\mathbf{q}^{\prime}, P^{\prime}, \theta, \phi, \rho^{\prime}$ are the perturbed quantity in velocity, pressure, the temperature of the fluid phase, the temperature of the solid phase, and density respectively. Now, the following non-linear system of perturbation equations (after dropping prime) is obtained:

$$
\begin{gathered}
\frac{\rho_{f}}{\varepsilon} \frac{\partial \mathbf{q}}{\partial t}=-\nabla P-\frac{\mu_{f}}{K} \mathbf{q}+\mu_{f}^{\prime} \nabla^{2} \mathbf{q}+\rho_{f} \alpha \theta \mathbf{g} \hat{k} \\
\nabla \cdot \mathbf{q}=0 \\
\varepsilon \frac{\partial \theta}{\partial t}+\mathbf{q} \cdot \nabla \theta=-\frac{d T_{b}}{d z} w+\varepsilon \kappa \nabla^{2} \theta+\frac{h}{(\rho c)_{f}}(\phi-\theta) \\
(1-\varepsilon)(\rho c)_{s} \frac{\partial \phi}{\partial t}=(1-\varepsilon) k_{s} \nabla^{2} \phi-h(\phi-\theta)
\end{gathered}
$$

In order to analyze the stability of the above system, we first reduce the complexity of the system by using the following scaling parameter,

$$
\begin{aligned}
& z=d z^{\star}, \mathbf{q}=\frac{\varepsilon k_{f}}{(\rho c)_{f} d} \mathbf{q}^{\star}, P=\frac{\mu_{f} \varepsilon k_{f}}{(\rho c)_{f} K} P^{\star}, \\
& t=\frac{d^{2}(\rho c)_{f}}{k_{f}} t^{\star}, H=\frac{h d^{2}}{\varepsilon k_{f}} \text {, } \\
& \theta=\frac{Q_{f} d^{2}(\rho c)_{f}}{k_{f} \sqrt{R a}} \theta^{\star}, \quad \phi=\frac{Q_{f} d^{2}(\rho c)_{f}}{k_{f} \sqrt{R a}} \phi^{\star}, \\
& V a=\frac{\varepsilon \mu_{f} c_{f} d^{2}}{k_{f} K}, D a=\frac{K}{d^{2}}, \\
& A=\frac{(\rho c)_{s} k_{f}}{(\rho c)_{f} k_{s}}, \quad \tilde{D} a=\frac{\mu_{f}^{\prime} K}{\mu_{f} d^{2}}, \\
& R a=\frac{\rho_{f} \alpha g Q_{f} K d^{3}}{\mu_{f} \varepsilon \kappa^{2}}, \quad \psi=\frac{\varepsilon k_{f}}{(1-\varepsilon) k_{s}}
\end{aligned}
$$

here, $H$ is inter-phase heat transfer parameter, $V a$ is Vadasz number, $D a$ is Darcy number, $A$ is diffusivity ratio, $\tilde{D} a$ is Darcy-Brinkman number, $R a$ is Darcy-Rayleigh number, and $\psi$ is porosity-modified conductivity ratio. 
Now, the system of non-dimensional perturbed equation (after dropping ${ }^{\star}$ ) can be written as:

$$
\begin{gathered}
\frac{1}{V a} \frac{\partial \mathbf{q}}{\partial t}=-\nabla P-\mathbf{q}+\tilde{D} a \nabla^{2} \mathbf{q}+\sqrt{R a} \theta \hat{k} \\
\nabla \cdot \mathbf{q}=0 \\
\frac{\partial \theta}{\partial t}+\mathbf{q} \cdot \nabla \theta=\frac{1}{\varepsilon} m(z) \sqrt{R a} w+\nabla^{2} \theta+H(\phi-\theta) \\
A \frac{\partial \phi}{\partial t}=\nabla^{2} \phi-\psi H(\phi-\theta)
\end{gathered}
$$

where

$$
m(z)=\left\{\begin{array}{l}
H_{1} z-H_{2} \xi \\
\frac{H_{1}}{2}\left(z^{2}+z\right)-H_{2} \xi \\
H_{1}\left(\frac{1}{2} z^{3}-\frac{3}{2} z^{2}+2 z\right)-H_{2} \xi \\
H_{1}\left(z-\frac{1}{2 \pi} \cos 2 \pi z-\frac{1}{4 \pi} \cos 4 \pi z+\frac{3}{4 \pi}\right) \\
-H_{2} \xi
\end{array}\right.
$$

case $A$ case $B$ case $C$

here, $H_{1}=\operatorname{sign}\left(Q_{f}\right), H_{2}=\operatorname{sign}(y)$, and $\xi=\frac{|y| \varepsilon \kappa}{\left|Q_{f}\right| d}$ is the heat parameter and the following are the corresponding boundaries for the above system of equation:

$$
\begin{gathered}
\mathbf{q}=\mathbf{0} \text { on } z=0,1 \\
\Theta=0, \Phi=0 \text { on } z=1 \\
D \Theta=0, D \Phi=0 \text { on } z=0
\end{gathered}
$$

\section{Stability Analysis}

For a stability analysis, there are mainly two techniques that may be employed, one is the method of linearized instability and another is the energy method. Infinitesimal small perturbations are assumed to the steady state, in linear analysis and so the impact of nonlinear terms becomes negligible and hence discarded from the set of partial differential equations. The linear theory only provides boundaries for instability; thus it is highly desirable to develop the nonlinear energy stability theory. For obtaining optimal nonlinear thresholds the idea of coupling parameter (Joseph 1965) can be employed for constructing energy functional. It will provide sharpen nonlinear thresholds as compared to the classical approach.

\subsection{Linear Analysis}

Non-linear terms are discarded from nondimensional system of the perturbed equation (13) - (16) and normal modes $(\mathbf{q}, \theta, \phi)=$ $[\mathbf{q}(z), \Theta(z), \Phi(z)] \exp \left[\sigma t+i\left(a_{x} x+a_{y} y\right)\right], \quad$ [Chandrasekhar 1981] introduced to tile the plane $(x, y)$. Here $\sigma t$ represents the temporal growth rate and $a_{x}, a_{y}$ shows the horizontal disturbance in the fluid layer. Considering $D=\frac{d}{d z}$ and to remove the pressure term operate curlcurl on (13), and the $\hat{\mathbf{k}}$ component is taken. So, we have the following linearized system:

$\frac{\sigma}{V a}\left(D^{2}-a^{2}\right) W=\tilde{D} a\left(D^{2}-a^{2}\right)^{2} W-\left(D^{2}-a^{2}\right) W-\sqrt{R a} a^{2} \Theta$

$$
\begin{gathered}
\sigma \Theta=\frac{\sqrt{R a}}{\varepsilon} m(z) W+\left(D^{2}-a^{2}\right) \Theta+H(\Phi-\Theta) \\
\sigma A \Phi=\left(D^{2}-a^{2}\right) \Phi-\psi H(\Phi-\Theta)
\end{gathered}
$$

Boundary conditions corresponding to the above system are given below [Straughan 2015]:

$$
W=0, D W=0, \quad \text { on } z=0,1
$$

$$
\Theta=0, \Phi=0 \text { on } z=1
$$

$$
D \Theta=0, D \Phi=0 \text { on } z=0
$$

For numerical analysis, Chebyshev-pseudospectral method is used. It an efficient numerical technique to find out eigenvalues and eigenfunctions in the system of continuum mechanics. The key point of this method is, one can calculate as many eigenvalues as required, not just the dominant one. Following the method laid out in Kaloni \& Lou (2004), the domain of the method is $[-1,1]$ but the present domain is $[0,1]$, so a simple transformation is used to match the domain of the method i.e., $\mathrm{z}$ is transformed into 2z-1. After transformation the system of linearized equations is:

$$
\begin{aligned}
& \frac{\sigma}{V a}\left(4 D^{2}-a^{2}\right) W=\tilde{D} a\left(4 D^{2}-a^{2}\right)^{2} W-\left(4 D^{2}-a^{2}\right) W \\
& -\sqrt{R a} a^{2} \Theta \\
& \sigma \Theta=\frac{\sqrt{R a}}{\varepsilon} n(z) W+\left(4 D^{2}-a^{2}\right) \Theta+H(\Phi-\Theta) \\
& \sigma A \Phi=\left(4 D^{2}-a^{2}\right) \Phi-\psi H(\Phi-\Theta)
\end{aligned}
$$


where

$$
n(z)= \begin{cases}H_{1}\left(\frac{z+1}{2}\right)-H_{2} \xi & \text { case } A \\ \frac{H_{1}}{2}\left(\frac{z+1}{2}\right)\left(\frac{z+3}{2}\right)-H_{2} \xi & \text { case } B \\ H_{1}\left[(z+1)-\frac{3}{2}\left(\frac{z+1}{2}\right)^{2}+\frac{1}{2}\left(\frac{z+1}{2}\right)^{3}\right]-H_{2} \xi & \text { case } C \\ H_{1}\left[\left(\frac{z+1}{2}\right)+\frac{1}{2 \pi} \cos \pi z-\frac{1}{4 \pi} \cos 2 \pi z+\frac{3}{4 \pi}\right] & \\ -H_{2} \xi & \text { case } D\end{cases}
$$

Next, discretizing equations (27) - (29) following the Chebyshev scheme and hence can be written in form of a system of the matrix as:

$$
\sigma \mathbf{A}_{\mathbf{1}} \mathbf{X}=\mathbf{A}_{\mathbf{2}} \mathbf{X}
$$

where $\mathbf{A}_{\mathbf{1}}$ and $\mathbf{A}_{\mathbf{2}}$ are complex matrices, given as:

$$
\mathbf{A}_{\mathbf{1}}=\left[\begin{array}{ccc}
\frac{1}{V a}\left(4 D^{2}-a^{2} I\right) & O & O \\
O & I & O \\
O & O & A I
\end{array}\right]
$$

and

$$
\mathbf{A}_{\mathbf{2}}=\left[\begin{array}{ccc}
\tilde{D} a\left(4 D^{2}-a^{2} I\right)^{2}-\left(4 D^{2}-a^{2} I\right) & -\sqrt{R a} a^{2} I & O \\
\frac{\sqrt{R a}}{\varepsilon} n(Z) & \left(4 D^{2}-a^{2} I\right)-H I & H I \\
O & \psi H I & \left(4 D^{2}-a^{2} I\right)-\psi H I
\end{array}\right]
$$

here $I$ and $O$ are identity and zero matrices. Results obtained by numerical simulation for the linear instability are presented in section 4 .

\subsection{Nonlinear stability analysis}

Adopting the standard nonlinear energy approach in the stability measure, we first multiply eqs. (13) - (16) with q, $\theta, \phi$ respectively and integrated over $V$ to have:

$$
\begin{gathered}
\frac{1}{2 V a} \frac{d}{d t}\|\mathbf{q}\|^{2}=\sqrt{R a}\langle w \theta\rangle-\|\mathbf{q}\|^{2}-\tilde{D} a\|\nabla \mathbf{q}\|^{2} \\
\frac{1}{2} \frac{d}{d t}\|\theta\|^{2}=\frac{\sqrt{R a}}{\varepsilon}\langle m(z) w \theta\rangle-\|\nabla \theta\|^{2}+H\langle\theta \phi\rangle-H\|\theta\|^{2} \\
\frac{A}{2} \frac{d}{d t}\|\phi\|^{2}=-\|\nabla \phi\|^{2}-\psi H\|\phi\|^{2}+\psi H\langle\phi \theta\rangle
\end{gathered}
$$

To attain steady states (5) nonlinear stability, the energy functional, formed by thermal energy and kinetic energy of perturbations of fluid and solid phase, using coupling parameters $\lambda_{1}$ and $\lambda_{2}$, is defined as given below:

$$
E(t)=\frac{1}{2}\|\theta\|^{2}+\frac{\lambda_{1} A}{2}\|\phi\|^{2}+\frac{\lambda_{2}}{2 V a}\|\mathbf{q}\|^{2}
$$

Variation in energy functional with respect to variation in time is given by:

$$
\frac{d}{d t} E(t)=\frac{1}{2} \frac{d}{d t}\|\theta\|^{2}+\frac{\lambda_{1} A}{2} \frac{d}{d t}\|\phi\|^{2}+\frac{\lambda_{2}}{2 V a} \frac{d}{d t}\|\mathbf{q}\|^{2}
$$

Using equations (32) - (34) in (36), we get

$$
\frac{d}{d t} E(t)=I-D
$$

where $I$ represents the energy production terms in the system and has the form

$$
I=\frac{\sqrt{R a}}{\epsilon}\langle m(z) w \theta\rangle+\lambda_{2} \sqrt{R a}\langle w \theta\rangle+H\langle\phi \theta\rangle+\lambda_{1} \psi H\langle\phi \theta\rangle
$$


and $D$ represents the energy dissipating terms that are as follows:

$$
\begin{aligned}
& D=\|\nabla \theta\|^{2}+H\|\theta\|^{2}+\lambda_{1}\|\nabla \phi\|^{2}+\psi H \lambda_{1}\|\phi\|^{2}+\lambda_{2}\|\mathbf{q}\|^{2} \\
& +\tilde{D} a \lambda_{2}\|\nabla \mathbf{q}\|^{2}
\end{aligned}
$$

Now, put

$$
\Re=\max _{\Omega} \frac{I}{D},
$$

where $\Omega$ being admissible solution space, such as $\Omega=$ $\left\{\mathbf{q}, \theta, \phi \mid \mathbf{q}, \theta, \phi \in L^{2}[0,1]\right\}$. Let, $\beta=1-\Re$, from the equation (37) it obtains:

$$
\frac{d}{d t} E(t) \leq-\beta D
$$

Next, using Poincaré's inequality and definition of $D$, we have the following inequality:

$$
\begin{aligned}
& D \geq\left(H+\pi^{2}\right)\|\theta\|^{2}+\left(\psi H+\pi^{2}\right) \lambda_{1}\|\phi\|^{2} \\
& +\left(1+\tilde{D} a \pi^{2}\right) \lambda_{2}\|\mathbf{q}\|^{2}
\end{aligned}
$$

Combining equations (41) and(42), one shows that for a constant $k>0$

$$
\frac{d}{d t} E(t) \leq-\beta k E(t)
$$

where $k=\min \left[2\left(H+\pi^{2}\right) ; 2\left(\frac{H+\pi^{2}}{A}\right) ; 2 V a\left(1+\tilde{D} a \pi^{2}\right)\right]$.

Equation (43) implies that $E(t) \leq E(0) \exp (-\beta k t)$ which shows that $E$ decays exponentially, yielding the nonlinear stability for $\theta, \phi$ and $\mathbf{q}$.

To obtain nonlinear stability bounds, the variational problem (40) must be solved. Therefore consider $\Re=1$, because it provides the nonlinear stability thresholds so the Euler-Lagrange equations for the maximization problem (40), after using rescaling $\left(\phi=\frac{1}{\sqrt{\lambda_{1}}} \phi, \mathbf{q}=\frac{1}{\sqrt{\lambda_{2}}} \mathbf{q}\right)$, are

$$
\begin{gathered}
2 \mathbf{q}-2 \tilde{D} a \nabla^{2} \mathbf{q}-\frac{\sqrt{R a}}{\varepsilon \sqrt{\lambda_{2}}} m(z) \theta \hat{\mathbf{k}}-\sqrt{\lambda_{2} R a} \theta \hat{\mathbf{k}}=2 \nabla s \\
-2 \nabla^{2} \theta+2 H \theta-\frac{\sqrt{R a}}{\varepsilon \sqrt{\lambda_{2}}} m(z) w-\sqrt{\lambda_{2} R a} w \\
-\frac{H}{\sqrt{\lambda_{1}}}\left(1+\psi \lambda_{1}\right) \phi=0 \\
-2 \nabla^{2} \phi+2 \psi H \phi-\frac{H}{\sqrt{\lambda_{1}}}\left(1+\psi \lambda_{1}\right) \theta=0
\end{gathered}
$$

as $\mathbf{q}$ is solenoidal so Lagrange multiplier $s$ is introduced. Next, we operate curlcurl on (44) and consider $\hat{\mathbf{k}}$ component, we have

$$
-2 \nabla^{2} w+2 \tilde{D} a \nabla^{4} w+\frac{\sqrt{R a}}{\varepsilon \sqrt{\lambda_{2}}} m(z) \nabla_{1}^{2} \theta+\sqrt{\lambda_{2} R a} \nabla_{1}^{2} \theta=0
$$

Next, the following tiling form is introduced to tile the plane:

$$
(w, \theta, \phi)=[W(z), \Theta(z), \Phi(z)] \exp \left[i\left(a_{x} x+a_{y} y\right)\right]
$$

So, from equations (45), (46) and (47) we have the following system of equations:

$$
\begin{aligned}
& 2\left(D^{2}-a^{2}\right) W-2 \tilde{D} a\left(D^{2}-a^{2}\right)^{2} W+\frac{\sqrt{R a}}{\varepsilon \sqrt{\lambda_{2}}} m(z) a^{2} \Theta \\
& +\sqrt{\lambda_{2} R a} a^{2} \Theta=0 \\
& \frac{\sqrt{R a}}{\varepsilon \sqrt{\lambda_{2}}} m(z) W+\sqrt{\lambda_{2} R a} W+2\left(D^{2}-a^{2}\right) \Theta-2 H \Theta \\
& +\frac{H}{\sqrt{\lambda_{1}}}\left(1+\psi \lambda_{1}\right) \Phi=0 \\
& \frac{H}{\sqrt{\lambda_{1}}}\left(1+\psi \lambda_{1}\right) \Theta+2\left(D^{2}-a^{2}\right) \Phi-2 \psi H \Phi=0
\end{aligned}
$$

and boundary conditions correspond to the above system are as follows:

$$
\begin{gathered}
W=0, D W=0, \text { on } z=0,1 \\
\Theta=0, \Phi=0 \text { on } z=1 \\
D \Theta=0, D \Phi=0 \text { on } z=0
\end{gathered}
$$

Now, following the method explained for linear analysis, the domain is transformed $[0,1]$ to $[-1,1]$. Now, the system of equations (49) - (54) forms an eigenvalue problem of order eight and it is utilized to localize the nonlinear critical Darcy-Rayleigh number $R a_{E}$, given by $R a=\max _{\lambda_{1}, \lambda_{2}} \min _{a} R a\left(\lambda_{1}, \lambda_{2}, a\right)$. Results derived by Chebyshev method are presented in section 4 .

\section{Result and Discussion}

The onset of convection in a Darcy-Brinkman porous medium is investigated for a local thermal nonequilibrium model in the presence of internal heat source. The various heat generating functions are taken into consideration which induces instability within the fluid layer when the heat is extracted through the boundary at a constant rate. Stability analysis performed by normal mode technique and energy method and Chebyshev-pseudospectral scheme used to solve generalized eigenvalue problem. A comparison of critical 

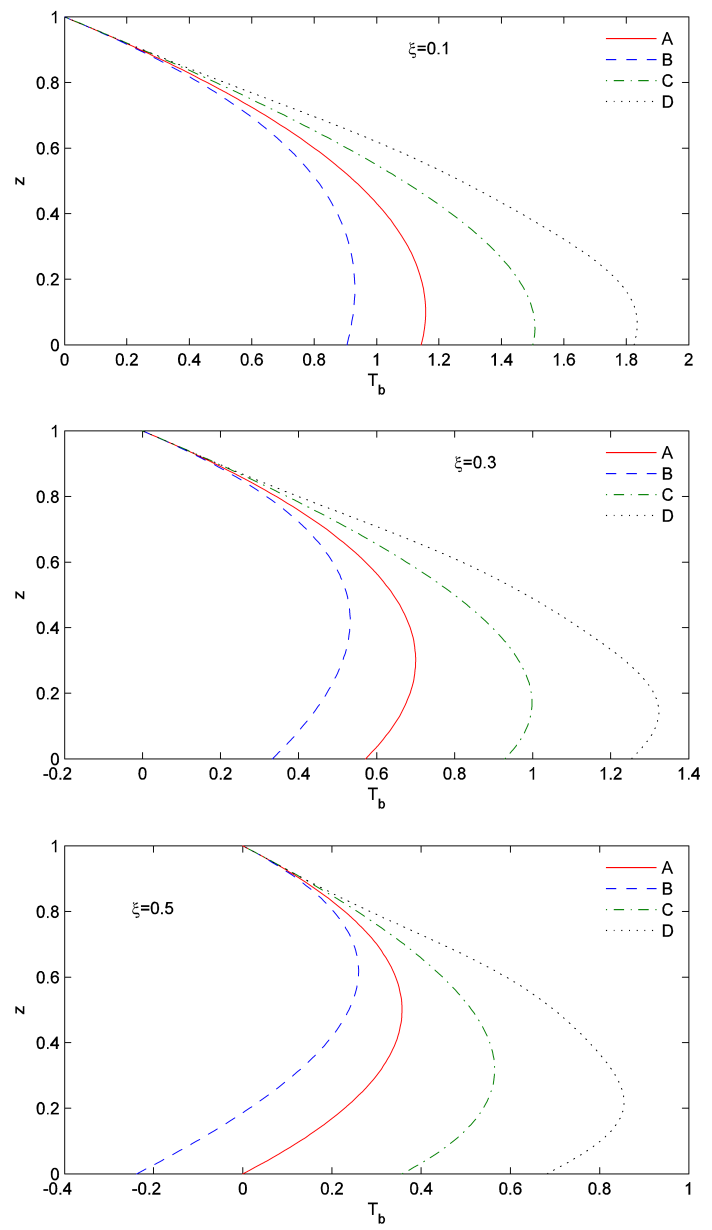

Fig. 2: Basic temperature profiles $T_{b}$ with fluid layer $z$

values of Darcy-Rayleigh number and wave number with respect to various parameters is performed for all heat generating functions and corresponding results depicted numerically and graphically. For the validation of the results, the model is reduced to the LTE limits and in the absence of heat extraction through the lower boundary the results are compared with the existing results and are presented in Table 1. It can be pointed out here that the obtained results appear with the multiple of an additional factor $1 / \varepsilon$, it is because the Rayleigh number is defined in terms of thermal diffusivity $\kappa=k_{f} /(\rho c)_{f}$ of the fluid rather than in terms of $\left[\varepsilon k_{f}+(1-\varepsilon) k_{s}\right] /(\rho c)_{f}$ [Kuznetsov \& Nield (2010)].

We start the discussion in the context of heat parameter $\xi$ ranges between $(0,1)$ for all heat supply functions and provide facts and figures concerning penetrative convection. From the temperature profile presented in Figure 2, it can be seen that lower part of the fluid layer has a high temperature for small values of $\xi$ (heat parameter) but as $\xi$ increases, the upper part of the fluid layer

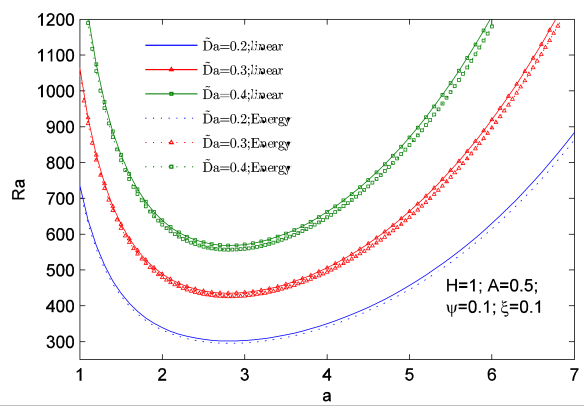

(A)

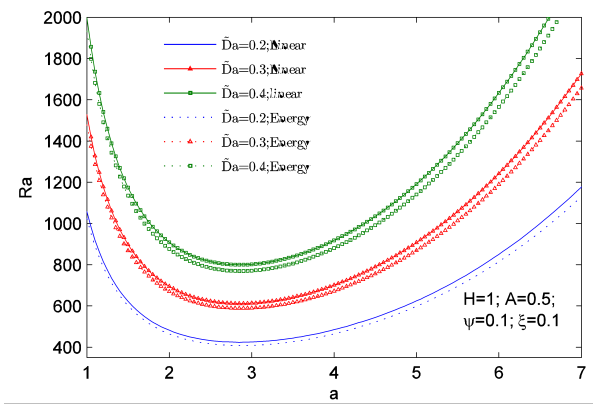

(B)

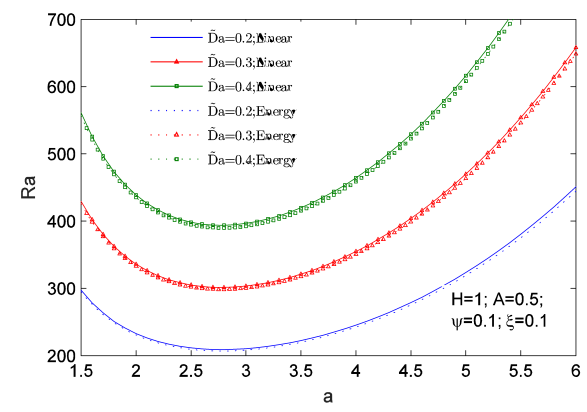

(C)

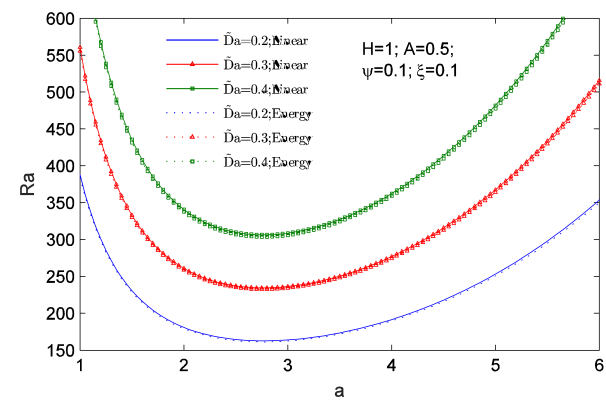

(D)

Fig. 3: Neutral stability curves for case $A, B, C$ and $D$ at different values of $\tilde{D} a$ and fixed values of $H, \xi, \psi, A$ 
Table 1: Comparison of the present study with the existing results.

\begin{tabular}{ccc}
\hline $\begin{array}{c}\text { Internal Heat } \\
\text { Parameter }\end{array}$ & Present Study & Existing Results \\
\hline$\xi=0$ & $R a_{L} / \varepsilon=471.385$ (for case $\left.\mathrm{A}\right)$ & $R a_{L}=471.38466$ [Borujerdi et al. (2008)] \\
\hline$\xi \gg 1$ & $R a_{L} / \varepsilon=39.237$ (for case $\left.\mathrm{A}\right)$ & $R a_{L}=39.478$ [Nield and Bejan (2013)] \\
\hline
\end{tabular}

Table 2: variation of critical Rayleigh number $\left(R a_{L}, R a_{E}\right)$ with internal heat parameter $(\xi)$ at fixed values of $\tilde{D} a, H, A, \psi$ for case A of the constant heat source

\begin{tabular}{|c|c|c|c|c|c|c|c|c|c|}
\hline & \multicolumn{5}{|c|}{$H=1 ; A=0.5 ; \psi=0.1$} & \multicolumn{4}{|c|}{$H=10 ; A=0.5 ; \psi=0.1$} \\
\hline \multirow{7}{*}{$\tilde{D} a=0$} & $\xi$ & $a_{c L}$ & $R a_{L}$ & $a_{c E}$ & $R a_{E}$ & $a_{c L}$ & $R a_{L}$ & $a_{c E}$ & $R a_{E}$ \\
\hline & 0.10 & 2.72 & 30.22 & 2.72 & 29.09 & 3.60 & 45.22 & 3.59 & 43.71 \\
\hline & 0.20 & 2.86 & 41.06 & 2.83 & 38.58 & 3.76 & 59.05 & 3.71 & 56.19 \\
\hline & 0.30 & 3.22 & 60.93 & 3.06 & 55.03 & 4.07 & 82.03 & 3.92 & 76.40 \\
\hline & 0.40 & 3.93 & 98.10 & 3.50 & 85.73 & 4.60 & 122.35 & 4.28 & 111.67 \\
\hline & 0.50 & 4.79 & 169.00 & 4.20 & 147.00 & 5.33 & 198.68 & 4.87 & 179.10 \\
\hline & 0.60 & 5.94 & 327.29 & 5.10 & 283.86 & 6.41 & 365.93 & 5.78 & 326.32 \\
\hline \multirow{6}{*}{$\tilde{D} a=0.2$} & 0.10 & 2.82 & 301.67 & 2.82 & 295.33 & 3.38 & 457.06 & 3.38 & 446.72 \\
\hline & 0.20 & 2.86 & 409.07 & 2.87 & 394.09 & 3.41 & 609.66 & 3.42 & 586.65 \\
\hline & 0.30 & 2.98 & 626.06 & 2.97 & 579.65 & 3.50 & 902.22 & 3.49 & 838.23 \\
\hline & 0.40 & 3.34 & 1199.58 & 3.21 & 999.19 & 3.76 & 1601.82 & 3.66 & 1369.13 \\
\hline & 0.50 & 4.20 & 2965.67 & 3.68 & 2146.54 & 4.44 & 3596.90 & 3.99 & 2713.49 \\
\hline & 0.60 & 5.16 & 8637.22 & 4.01 & 5122.76 & 5.38 & 9885.04 & 4.22 & 6120.99 \\
\hline \multirow{6}{*}{$\tilde{D} a=0.4$} & 0.10 & 2.81 & 568.46 & 2.82 & 556.66 & 3.36 & 862.58 & 3.37 & 843.22 \\
\hline & 0.20 & 2.86 & 770.98 & 2.86 & 743.03 & 3.40 & 1151.16 & 3.40 & 1107.91 \\
\hline & 0.30 & 2.97 & 1181.01 & 2.96 & 1093.97 & 3.48 & 1706.06 & 3.47 & 1584.97 \\
\hline & 0.40 & 3.32 & 2274.90 & 3.19 & 1891.55 & 3.73 & 3045.34 & 3.63 & 2596.78 \\
\hline & 0.50 & 4.18 & 5711.08 & 3.67 & 4092.49 & 4.41 & 6930.60 & 3.96 & 5178.03 \\
\hline & 0.60 & 5.14 & 16842.82 & 3.98 & 9780.54 & 5.36 & 19282.93 & 4.18 & 11700.66 \\
\hline
\end{tabular}

Table 3: variation of critical Rayleigh number $\left(R a_{L}, R a_{E}\right)$ with internal heat parameter $(\xi)$ at fixed values of $\tilde{D} a, H, A, \psi$ for case B of linearly increasing heat generation

\begin{tabular}{|c|c|c|c|c|c|c|c|c|c|}
\hline & \multicolumn{5}{|c|}{$H=1 ; A=0.5 ; \psi=0.1$} & \multicolumn{4}{|c|}{$H=10 ; A=0.5 ; \psi=0.1$} \\
\hline \multirow{7}{*}{$\tilde{D} a=0$} & $\xi$ & $a_{c L}$ & $R a_{L}$ & $a_{c E}$ & $R a_{E}$ & $a_{c L}$ & $R a_{L}$ & $a_{c E}$ & $R a_{E}$ \\
\hline & 0.10 & 2.85 & 41.86 & 2.84 & 39.30 & 3.77 & 60.23 & 3.72 & 57.11 \\
\hline & 0.20 & 3.24 & 62.65 & 3.09 & 56.29 & 4.13 & 83.90 & 3.96 & 77.65 \\
\hline & 0.30 & 4.04 & 100.82 & 3.57 & 87.43 & 4.73 & 124.37 & 4.38 & 112.63 \\
\hline & 0.40 & 4.98 & 169.12 & 4.35 & 146.12 & 5.54 & 196.60 & 5.02 & 175.92 \\
\hline & 0.50 & 6.17 & 305.92 & 5.42 & 264.67 & 6.65 & 339.42 & 5.99 & 301.07 \\
\hline & 0.60 & 7.95 & 623.80 & 5.70 & 465.49 & 8.34 & 666.12 & 6.42 & 532.86 \\
\hline \multirow{6}{*}{$\tilde{D} a=0.2$} & 0.10 & 2.86 & 424.26 & 2.87 & 408.00 & 3.42 & 632.54 & 3.42 & 606.97 \\
\hline & 0.20 & 2.99 & 661.34 & 2.99 & 608.22 & 3.51 & 951.00 & 3.51 & 876.73 \\
\hline & 0.30 & 3.41 & 1309.32 & 3.25 & 1067.32 & 3.82 & 1730.71 & 3.69 & 1450.40 \\
\hline & 0.40 & 4.34 & 3228.63 & 3.79 & 2293.02 & 4.58 & 3877.28 & 4.08 & 2868.81 \\
\hline & 0.50 & 5.32 & 8748.98 & 4.12 & 5324.85 & 5.55 & 9940.85 & 4.33 & 6303.40 \\
\hline & 0.60 & 6.86 & 29199.23 & 4.03 & 9264.56 & 7.04 & 31632.86 & 4.24 & 11000.73 \\
\hline \multirow{6}{*}{$\tilde{D} a=0.4$} & 0.10 & 2.86 & 799.74 & 2.87 & 769.41 & 3.40 & 1194.60 & 3.41 & 1146.56 \\
\hline & 0.20 & 2.98 & 1248.04 & 2.98 & 1148.33 & 3.49 & 1799.17 & 3.49 & 1658.56 \\
\hline & 0.30 & 3.39 & 2486.95 & 3.23 & 2022.54 & 3.79 & 3295.60 & 3.67 & 2753.86 \\
\hline & 0.40 & 4.32 & 6232.58 & 3.76 & 4380.16 & 4.55 & 7488.97 & 4.04 & 5484.60 \\
\hline & 0.50 & 5.31 & 17087.63 & 4.08 & 10187.41 & 5.53 & 19422.31 & 4.29 & 12078.41 \\
\hline & 0.60 & 6.85 & 57561.42 & 3.99 & 17686.43 & 7.03 & 62367.06 & 4.19 & 21026.02 \\
\hline
\end{tabular}


Table 4: Variation of critical Rayleigh number $\left(R a_{L}, R a_{E}\right)$ with internal heat parameter $(\xi)$ at fixed values of $\tilde{D} a, H, A, \psi$ for case $\mathrm{C}$ of decreasing heat source

\begin{tabular}{|c|c|c|c|c|c|c|c|c|c|}
\hline & \multicolumn{5}{|c|}{$H=1 ; A=0.5 ; \psi=0.1$} & \multicolumn{4}{|c|}{$H=10 ; A=0.5 ; \psi=0.1$} \\
\hline \multirow{7}{*}{$\tilde{D} a=0$} & $\xi$ & $a_{c L}$ & $R a_{L}$ & $a_{c E}$ & $R a_{E}$ & $a_{c L}$ & $R a_{L}$ & $a_{c E}$ & $R a_{E}$ \\
\hline & 0.10 & 2.63 & 20.98 & 2.64 & 20.59 & 3.48 & 32.47 & 3.47 & 31.90 \\
\hline & 0.20 & 2.68 & 25.99 & 2.68 & 25.27 & 3.53 & 39.56 & 3.52 & 38.61 \\
\hline & 0.30 & 2.76 & 33.92 & 2.75 & 32.47 & 3.62 & 50.25 & 3.59 & 48.53 \\
\hline & 0.40 & 2.94 & 47.80 & 2.87 & 44.52 & 3.80 & 67.67 & 3.72 & 64.33 \\
\hline & 0.50 & 3.39 & 74.69 & 3.13 & 66.93 & 4.14 & 98.68 & 3.95 & 91.95 \\
\hline & 0.60 & 4.11 & 130.02 & 3.61 & 114.12 & 4.68 & 160.27 & 4.33 & 146.91 \\
\hline \multirow{6}{*}{$\tilde{D} a=0.2$} & 0.10 & 2.78 & 208.98 & 2.78 & 207.02 & 3.35 & 321.04 & 3.35 & 317.78 \\
\hline & 0.20 & 2.79 & 256.27 & 2.80 & 252.71 & 3.36 & 391.05 & 3.36 & 385.30 \\
\hline & 0.30 & 2.82 & 330.70 & 2.83 & 323.32 & 3.38 & 499.23 & 3.38 & 487.77 \\
\hline & 0.40 & 2.88 & 463.79 & 2.88 & 444.98 & 3.42 & 686.59 & 3.42 & 659.32 \\
\hline & 0.50 & 3.03 & 758.31 & 3.00 & 692.35 & 3.53 & 1076.41 & 3.51 & 991.80 \\
\hline & 0.60 & 3.54 & 1659.52 & 3.29 & 1333.66 & 3.88 & 2149.49 & 3.70 & 1792.55 \\
\hline \multirow{6}{*}{$\tilde{D} a=0.4$} & 0.10 & 2.77 & 393.75 & 2.78 & 390.09 & 3.33 & 605.63 & 3.34 & 599.53 \\
\hline & 0.20 & 2.79 & 482.82 & 2.80 & 476.21 & 3.34 & 737.75 & 3.35 & 727.00 \\
\hline & 0.30 & 2.82 & 623.03 & 2.82 & 609.31 & 3.37 & 942.01 & 3.37 & 920.55 \\
\hline & 0.40 & 2.87 & 873.94 & 2.88 & 838.85 & 3.41 & 1296.26 & 3.41 & 1244.94 \\
\hline & 0.50 & 3.02 & 1430.70 & 2.99 & 1306.65 & 3.51 & 2035.89 & 3.49 & 1875.24 \\
\hline & 0.60 & 3.52 & 3156.21 & 3.29 & 2526.64 & 3.85 & 4095.24 & 3.68 & 3401.07 \\
\hline
\end{tabular}

Table 5: Variation of critical Rayleigh number $\left(R a_{L}, R a_{E}\right)$ with internal heat parameter $(\xi)$ at fixed values of $\tilde{D} a, H, A, \psi$ for case D of nonuniform heat generation

\begin{tabular}{|c|c|c|c|c|c|c|c|c|c|}
\hline & & \multicolumn{4}{|c|}{$H=1 ; A=0.5 ; \psi=0.1$} & \multicolumn{4}{|c|}{$H=10 ; A=0.5 ; \psi=0.1$} \\
\hline \multirow{7}{*}{$\tilde{D} a=0$} & $\xi$ & $a_{c L}$ & $R a_{L}$ & $a_{c E}$ & $R a_{E}$ & $a_{c L}$ & $R a_{L}$ & $a_{c E}$ & $R a_{E}$ \\
\hline & 0.10 & 2.62 & 16.34 & 2.62 & 16.09 & 3.45 & 25.47 & 3.45 & 25.10 \\
\hline & 0.20 & 2.64 & 19.25 & 2.64 & 18.85 & 3.48 & 29.69 & 3.48 & 29.14 \\
\hline & 0.30 & 2.68 & 23.38 & 2.68 & 22.69 & 3.53 & 35.49 & 3.52 & 34.61 \\
\hline & 0.40 & 2.76 & 29.59 & 2.74 & 28.33 & 3.62 & 43.87 & 3.58 & 42.40 \\
\hline & 0.50 & 2.90 & 39.72 & 2.84 & 37.17 & 3.76 & 56.74 & 3.68 & 54.14 \\
\hline & 0.60 & 3.22 & 57.71 & 3.03 & 52.28 & 4.00 & 78.00 & 3.85 & 73.19 \\
\hline \multirow{6}{*}{$\tilde{D} a=0.2$} & 0.10 & 2.77 & 162.62 & 2.77 & 161.44 & 3.34 & 250.68 & 3.34 & 248.71 \\
\hline & 0.20 & 2.78 & 189.96 & 2.78 & 188.10 & 3.35 & 291.57 & 3.35 & 288.51 \\
\hline & 0.30 & 2.80 & 228.24 & 2.80 & 225.07 & 3.36 & 348.18 & 3.36 & 343.09 \\
\hline & 0.40 & 2.82 & 285.49 & 2.82 & 279.47 & 3.38 & 431.48 & 3.38 & 422.15 \\
\hline & 0.50 & 2.86 & 379.89 & 2.87 & 366.55 & 3.41 & 565.29 & 3.41 & 545.71 \\
\hline & 0.60 & 2.96 & 561.15 & 2.95 & 523.93 & 3.48 & 810.67 & 3.47 & 760.81 \\
\hline \multirow{6}{*}{$\tilde{D} a=0.4$} & 0.10 & 2.77 & 306.40 & 2.77 & 304.20 & 3.33 & 472.87 & 3.33 & 469.19 \\
\hline & 0.20 & 2.78 & 357.90 & 2.78 & 354.44 & 3.33 & 550.02 & 3.34 & 544.30 \\
\hline & 0.30 & 2.79 & 429.99 & 2.80 & 424.10 & 3.34 & 656.85 & 3.35 & 647.32 \\
\hline & 0.40 & 2.81 & 537.85 & 2.82 & 526.65 & 3.36 & 814.12 & 3.37 & 796.64 \\
\hline & 0.50 & 2.86 & 715.75 & 2.86 & 690.90 & 3.39 & 1066.99 & 3.40 & 1030.22 \\
\hline & 0.60 & 2.95 & 1057.91 & 2.95 & 988.20 & 3.46 & 1531.80 & 3.45 & 1437.56 \\
\hline
\end{tabular}

has high temperature thus the stabilizing effect of $\xi$ is expected. In Table 2-5, obtained values of critical DarcyRayleigh numbers by linear approach $\left(R a_{L}\right)$ and nonlinear approach $\left(R a_{E}\right)$ with variation in heat parameter $(\xi)$, Darcy-Brinkman number $(\tilde{D} a)$, at fixed values of porositymodified conductivity ratio $(\psi)$, inter-phase heat parameter $(H)$, diffusivity ratio $(A)$, are provided. It is found that for a Darcy model $(\tilde{D} a=0)$, as $\xi$ increases, the crit- ical value of Darcy-Rayleigh number increases. Increase in value of heat parameter adds on the stability of the system. The heat extraction through the lower boundary has a counter effect to the internal heat generation, which slows down the heat transfer and results in a delay in convection. The Darcy-Brinkman number has a significant influence on the stability of the system, as the value of $\tilde{D} a$ increases, a substantial change occurs in the value of the critical 


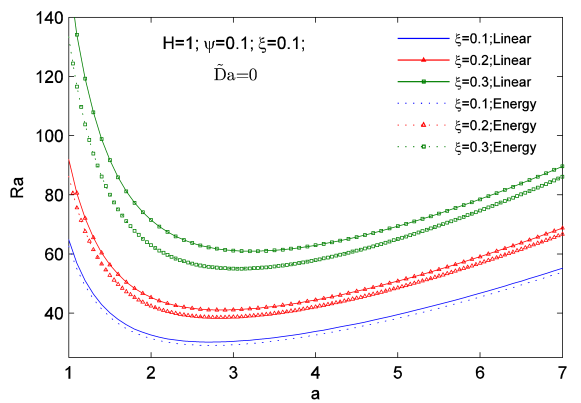

(A)

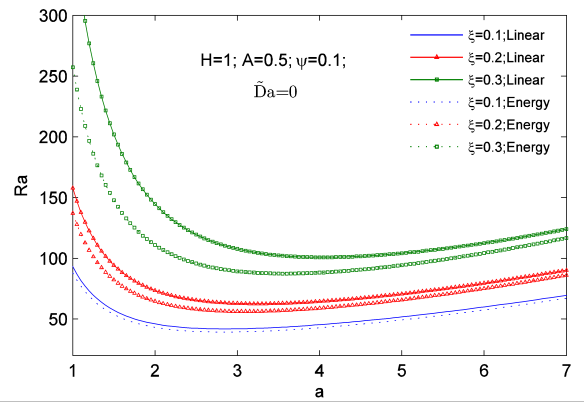

(B)

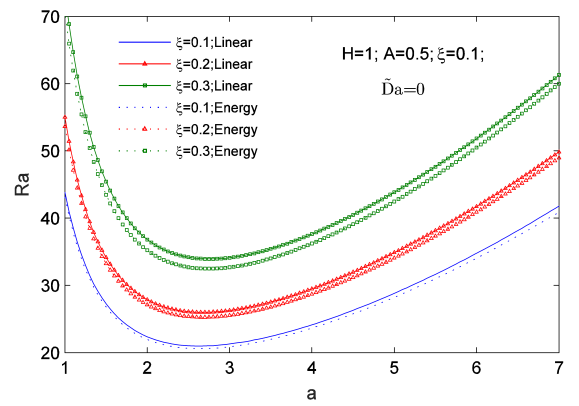

(C)

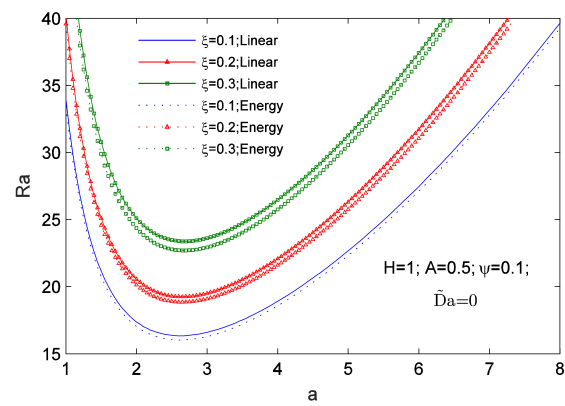

(D)

Fig. 4: Neutral stability curves for case A, B, C and D at different values of $\xi$ and fixed values of $H, \psi, \tilde{D} a, A$

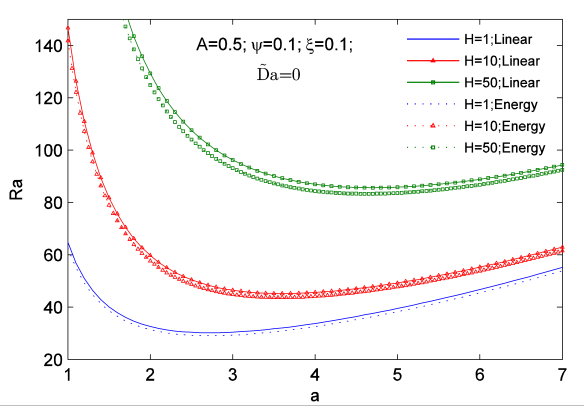

(A)

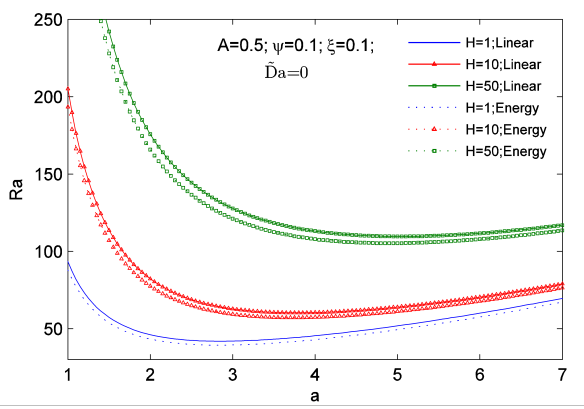

(B)

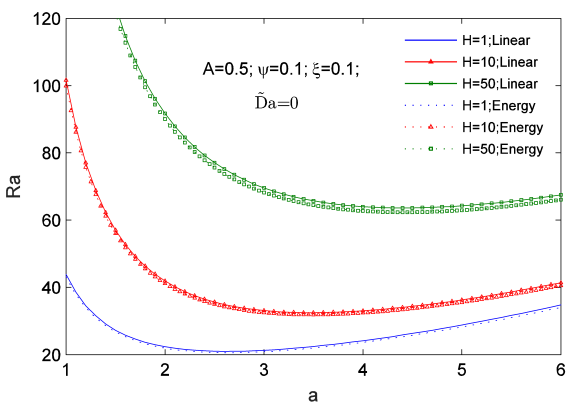

(C)

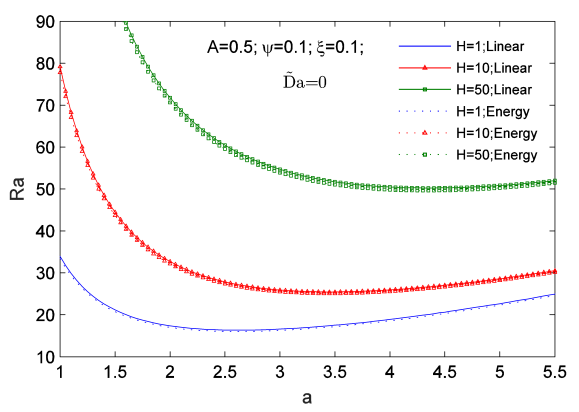

(D)

Fig. 5: Neutral stability curves for case A, B, C and D at different values of $H$ and fixed values of $\psi, \xi, \tilde{D} a, A$ 


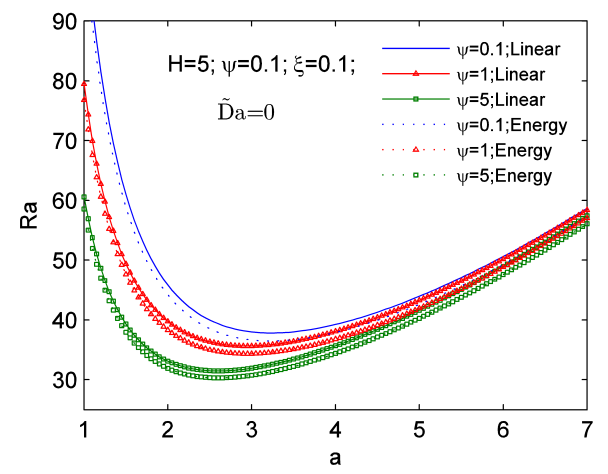

(A)

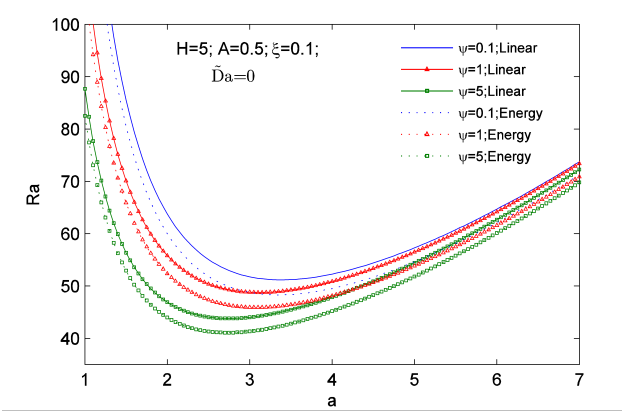

(B)

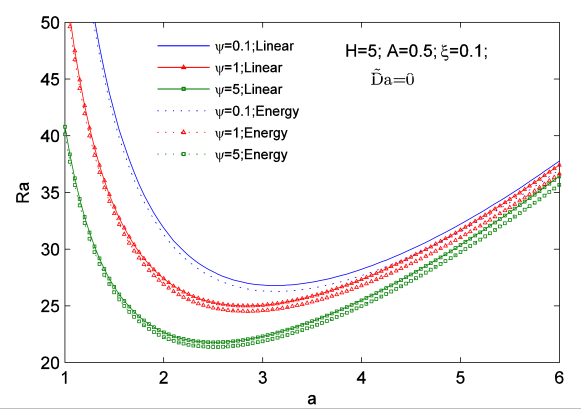

(C)

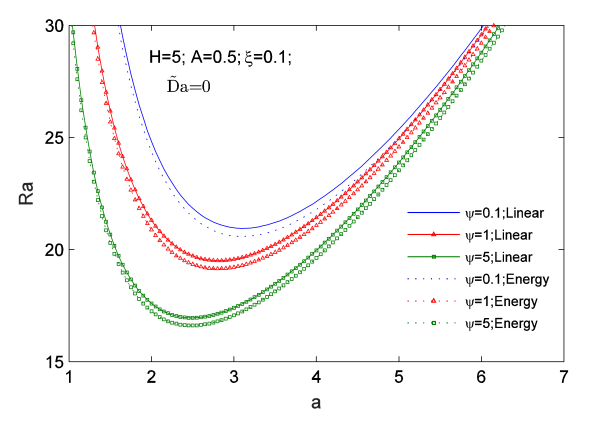

(D)

Fig. 6: Neutral stability curves for case A, B, C and D at different values of $\psi$ and fixed values of $H, \xi, \tilde{D} a, A$
Rayleigh number for both linear and nonlinear analysis. Again, from tables, It is also observed that increase in the value of $H$, the value of the critical Rayleigh number increases and the onset of convection delays. Cell size gets contracted for heat supply functions for increasing values of $\xi$ and $H$.

The neutral stability curves for Rayleigh number and wave number for distinct parameters are shown in Figures 3-6. A neutral stability curve, in the expression of critical Darcy-Rayleigh number, provides the stability criterion. In the region above the linear plot, system's behavior is unstable and in the region below the nonlinear plot, its behavior is stable. These curves show that for a range of wave number the linear and nonlinear thresholds are so close that they are indistinguishable graphically, signifying the suitability of linear theory to predict the nature of the onset of convection. But after a certain range of wave number, nonlinear threshold leave a region of potential subcritical instability. Further, from figure 3 it is observed that as the value of Darcy-Brinkman number increases, quantitative change occurs in the value of the critical Rayleigh number but the qualitative behavior remains the same. Increase in $\tilde{D} a$ is related to increasing effective viscosity of the fluid that retards the fluid flow and hence convection delays. Figure 4, represented the neutral curves for changing values of $\xi$. From these, it is observed that as $\xi$ increases, the critical value of Darcy-Rayleigh number increases. Increase in value of $\xi$ leads to the system stable, the reason for this behavior can be understood from the figure 2 which shows that for the small values of $\xi$, system is more unstable in the lower parts of the fluid layer due to the large temperature difference across the layer but as the value of $\xi$ increases, the temperature difference across the layer decreases which stabilize the fluid layer. As a result, with increasing values of $\xi$, convection slows down.

The increase in the values of the inter-phase heat transfer parameter has a significant impact on the critical Rayleigh number as shown in Figure 5. For small values of $H$, no significant heat transfer takes place between the solid and fluid phase and hence the properties of the solid phase does not affect the convection and system is in a strong LTNE situation. Next, as $H$ increases, we found a noticeable change in the critical values of the Rayleigh number because the solid properties affect the onset of convcetion. Figure 6 represents that as the value of $\psi$ increases, the value of critical Rayleigh number decreases; this is because of the increase in the value of $\psi$ leads to substantial transfer of heat through the solid and fluid phases. For very small values of $H$, the critical Rayleigh number is independent of the values of $\psi$ and approaches the LTE results. On the other hand, for comparatively large 
values of $H$ (for graphical results the value of $H$ is taken equal to 5), a significant change in the values of critical Rayleigh number can be observed with the increasing values of $\psi$.

\section{Conclusion}

This work is a detail presentation of stability analysis of a fluid saturating porous medium by linear and nonlinear analyses for four heat supply functions within the fluid layer with heat extraction through the lower boundary. It is observed that the system is more stable at large values of heat parameter as compared to small values, i.e. higher the heat parameter, higher the stability of the system. The two theories (linear and nonlinear) do not show any resemblance in results, it implies the region of subcritical instability exists. For all heat generating functions, it is observed that cell size gets affected with variation in values heat parameter, Darcy-Brinkman number, and interphase heat transfer parameter. Increasing values of DarcyBrinkman number, heat parameter, and inter-phase heat transfer parameter delays the onset of convection so these parameters found to be having stabilizing effect on the system. The increase in the value of porosity-modified conductivity ratio hastens the onset of convection and has a destabilizing effect on the stability of the system.

\section{Nomenclature}

\author{
$A$ Diffusivity ratio \\ $a_{c}$ Critical wave number \\ $a_{c L}$ Critical wave number for linear analysis \\ $a_{c E}$ Critical wave number by nonlinear analysis \\ Da Darcy number \\ $\tilde{D} a$ Darcy-Brinkman number \\ $d$ The depth of the fluid layer \\ g Gravity \\ $H$ Inter-phase heat transfer parameter \\ $H_{1}$ Heat generation within the fluid layer \\ $\mathrm{H}_{2}$ Heat extraction through lower boundary \\ $K$ Permeability \\ $P$ Pressure \\ $Q_{f}$ Internal heat source for fluid phase \\ $Q_{s}$ Internal heat source for solid phase \\ q Filter velocity \\ $R a$ Rayleigh number \\ $R a_{L}$ Rayleigh number by linear analysis \\ $R a_{E}$ Rayleigh number by nonlinear analysis
}

$T_{b}$ Basic temperature

$T_{f}$ Fluid phase temperature

$T_{S}$ Solid phase temperature

$T_{U}$ Upper boundary temperature

$t$ Time

$V a$ Vadasz number

\section{Greek Symbols}

$\varepsilon$ Porosity

$\kappa$ Thermal diffusivity

$k_{s}$ Thermal conductivity of the solid phase

$\lambda_{1}, \lambda_{2}$ Coupling parameters

$\mu_{f}$ Dynamic viscosity of the fluid

$\mu_{f}^{\prime}$ Effective viscosity of the fluid

$\rho_{f}$ Density of fluid

$\rho_{s}$ Density of solid matrix

$\rho^{\prime}$ Perturbation in density

$\theta$ Perturbation in fluid phase temperature

$\phi$ Perturbation in solid phase temperature

$\psi$ Porosity-modified conductivity ratio

$\xi$ Heat parameter

$y$ Rate of heat extraction through lower boundary

\section{References}

[1] Banu, N., Rees, D.A.S.: Onset of Darcy-Bénard convection using a thermal non-equilibrium model, Int. J. Heat Mass Transfer, 2002, 45, 2221-2228.

[2] Brinkman, H.C.: A calculation of the viscous force exerted by a flowing fluid on a dense swarm of particles, Appl. Sci. Res., 1947, 1, 27-34.

[3] Borujerdi, A.N., Noghrehabadi, A.R. and Rees, D.A.S., Influence of Darcy number on the onset of convection in a porous layer with a uniform heat source, Int. J. Therm. Sci., 2008, 47, 1020-1025.

[4] Carr, M., Putter, S.D.: Penetrative convection in a horizontally isotropic porous layer, Continuum Mech. Thermodyn., 2003, 15, 33-43.

[5] Chandrasekhar, S.: Hydrodynamic and Hydromagnetic Stability, Dover, New York, 1981.

[6] Gasser, R.D., Kazimi, M.S.: Onset of convection in a porous medium with internal heat generation, J. Heat Tranfer., 1976, 98, 49-54.

[7] Hill, A.A.: Penetrative convection induced by the absorption of radiation with a nonlinear heat source, Dyn. Atmos. Oceans, 2004, 38, 57-67.

[8] Hill, A.A., Carr, M.: The influence of a fluid-porous interface on the solar pond stability, Advances in water resources, 2013, 52, 1-6.

[9] Hill, A.A., Carr, M.: Stabilizing solar ponds by utilizing porous material, Advances in water resources, 2013, 60, 1-6. 
[10] Joseph, D.D.: On the stability of Boussinesq equations, Arch. Rational Mech. Anal., 1965, 20, 59-71.

[11] Kaloni, P.N., Lou, J.X.: Convective instability of magnetic fluids, Phys. Rev. E, 2004, 70, 1-12.

[12] Krishnamurti, R.: Convection induced by selective absorption of radiation: a laboratory model of conditional instability, Dyn. Atmos. Oceans, 1997, 27, 367-382.

[13] Kuznetsov, A.V., Nield, D.A.: Effect of local thermal nonequilibrium on the onset of convection in a porous medium layer saturated by a nanofluid, Transp Porous Med., 2010, 83, 425-436.

[14] Larson, V.E.: Stability properties of and scaling laws for a dry radiative-convective atmosphere, Q.J.R. Met. Soc., 2000, 126, 145-171.

[15] Matthews, P.C.: A model for the onset of penetrative convection, J. Fluid Mech., 1988, 188, 571-583.

[16] Nandal, R., Mahajan, A.: Linear and nonlinear stability analysis of a Horton-Rogers-Lapwood problem with an internal heat source and Brinkman effects, Transp Porous Med, 2017, 117, 261-280.

[17] Nield, D.A., Bejan, A.: Convection in Porous Media, Springer, Berlin, 2013.

[18] Nield, D.A., Kuznetsov, A.V.: Local thermal non-equilibrium and heterogeneity effects on the onset of convection in a layered porous medium with vertical throughflow, J. Porous Med., 2015, 18, 125-136.

[19] Rees, D.A.S.: The onset of Darcy-Brinkman convection in a porous layer: an asymptotic analysis, Int. J. Heat Mass Transfer., 2002, 45, 2213-2220.
[20] Roberts, P.H.: Convection in horizontal layers with internal heat generation. Theory, J. Fluid Mech., 1967, 30, 33-49.

[21] Straughan, B.: Continuous dependence on the heat source and non-linear stability in penetrative convection, Int. J. NonLinear Mechanics, 1991, 26, 221-231.

[22] Straughan, B.: Convection caused by radiation through the layer, IMA J. Appl. Math., 1991, 46, 211-216.

[23] Straughan, B.: The Energy Method, Stability, and Nonlinear Convection. Springer, Berlin, 2004.

[24] Straughan, B.: Convection with Local Thermal Non-Equilibrium and Microfluidic Effects, Springer, 2015.

[25] Wang, P., Vafai, K., Liu, D.Y.: Analysis of radiative effect under local thermal non-equilibrium conditions in porous mediaapplication to a solar air receiver, Numer. Heat Transfer, Part A, 2014, 65, 931-948.

[26] Postelnicu, A.: The onset of Darcy-Brinkman convection using a thermal nonequilibrium model. Part II, Int. J. Therm. Sci, 2008, 47, 1587-1594.

[27] Sunil, Sharma, P., Mahajan, A.: Onset of Darcy-Brinkman double-diffusive convection in a magnetized ferrofluid layer using a thermal non-equilibrium model: a nonlinear stability analysis, J. Geophys. Eng., 2010, 7, 417-430.

[28] Shivakumara, I.S., Dhananjaya, M.: Penetrative Brinkman convection in an anisotropic porous layer saturated by a nanofluid, Ain Shams Engineering Journal, 2015, 6, 703-713.

[29] Umavathi, J.C., Sheremet, M.A.: Onset of double-diffusive convection of a sparsely packed micropolar fluid in a porous medium layer saturated with a nanofluid, Microfluid Nanofluidics, 2017, 21, 128. 